\title{
BMJ Open REACH: study protocol of a randomised trial of rehabilitation very early in congenital hemiplegia
}

Roslyn N Boyd, ${ }^{1}$ Jenny Ziviani, ${ }^{2,3}$ Leanne Sakzewski, ${ }^{1}$ Iona Novak, ${ }^{4}$ Nadia Badawi, ${ }^{4}$ Kerstin Pannek, ${ }^{5}$ Catherine Elliott, ${ }^{6,7}$ Susan Greaves, ${ }^{8}$ Andrea Guzzetta, ${ }^{9}$ Koa Whittingham, ${ }^{1}$ Jane Valentine, ${ }^{7}$ Cathy Morgan, ${ }^{4}$ Margaret Wallen, ${ }^{4,10}$ Ann-Christin Eliasson, ${ }^{11}$ Lisa Findlay, ${ }^{2}$ Robert Ware, ${ }^{12}$ Simona Fiori, ${ }^{9}$ Stephen Rose ${ }^{13}$

To cite: Boyd RN, Ziviani J, Sakzewski L, et al. REACH: study protocol of a randomised trial of rehabilitation very early in congenital hemiplegia. BMJ Open 2017;7:e017204. doi:10.1136/ bmjopen-2017-017204

- Prepublication history for this paper is available online. To view these files please visit the journal online (http://dx.doi org/10.1136/bmjopen-2017017204).

Received 7 April 2017 Revised 22 June 2017 Accepted 29 June 2017

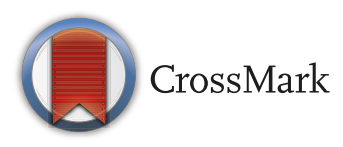

For numbered affiliations see end of article.

Correspondence to Professor Roslyn N Boyd; r.boyd@uq.edu.au

\section{ABSTRACT}

Objectives Congenital hemiplegia is the most common form of cerebral palsy (CP). Children with unilateral CP show signs of upper limb asymmetry by 8 months corrected age (ca) but are frequently not referred to therapy until after 12 months ca. This study compares the efficacy of infant-friendly modified constraint-induced movement therapy (Baby mCIMT) to infant friendly bimanual therapy (Baby BIM) on upper limb, cognitive and neuroplasticity outcomes in a multisite randomised comparison trial.

Methods and analysis 150 infants (75 in each group), aged between 3 and 6 months ca, with asymmetric brain injury and clinical signs of upper extremity asymmetry will be recruited. Children will be randomised centrally to receive equal doses of either Baby mCIMT or Baby BIM. Baby mCIMT comprises restraint of the unimpaired hand using a simple restraint (eg, glove, sock), combined with intensive parent implemented practice focusing on active use of the impaired hand in a play-based context. In contrast, Baby BIM promotes active play requiring both hands in a play-based context. Both interventions will be delivered by parents at home with monthly home visits and interim telecommunication support by study therapists. Assessments will be conducted at study entry; at 6, 12 months ca immediately postintervention (primary outcome) and 24 months ca (retention). The primary outcome will be the Mini-Assisting Hand Assessment. Secondary outcomes include the Bayley Scale for Infant and Toddler Development (cognitive and motor domains) and the Hand Assessment of Infants. A subset of children will undertake MRI scans at 24 months ca to evaluate brain lesion severity and brain (re)organisation after intervention.

Ethics and dissemination Full ethical approvals for this study have been obtained from the relevant sites. The findings will be disseminated in peer-reviewed publications.

Trial registration number Australian and New Zealand Clinical Trials Registry: ACTRN12615000180516, Pre results.

\section{INTRODUCTION}

Congenital hemiplegia occurs in over 1 million children under 21 years of age in the industrialised world. ${ }^{1}$ Congenital hemiplegia, frequently termed unilateral cerebral palsy (UCP), is characterised by a unilateral or asymmetric brain injury occurring around
Strengths and limitations of this study

- This study is an adequately powered randomised comparison trial of two alternate approaches to early intervention for infants at high risk of unilateral cerebral palsy.

- Primary outcomes are video recorded and assessed by a rater masked to group allocation.

- At 24 months (corrected age) brain (re)organisation will be compared for infants who receive alternate treatments.

- A controlled trial is not feasible or ethical.

- There are limited data published on variations in postural upper limb asymmetries and hand function for infants under 12 months of age with which to compare our data.

the time of birth, impacting development of hand skills and motor abilities on one side. Cerebral palsy is the fifth most costly health condition. $^{2}$ Therefore, early interventions that mitigate the brain injury and improve early hand function and later vocational and life outcomes are urgently needed. Children with congenital unilateral or asymmetric brain lesions frequently develop hemiplegia with major limitations in the use of their impaired hand, which results in poor bimanual coordination and impacts on the performance of daily activities in home, school and community life.

Currently, two very different intensive therapy approaches are used each with good evidence of improving outcomes for schoolaged children with CP. ${ }^{3}$ Traditional upper limb therapy adopts a bimanual approach (BIM) which aims to improve the use of the impaired hand as an assisting hand in play and functional daily activities. ${ }^{4}$ More recently, modified constraint-induced movement therapy (mCIMT) has been introduced, 
which employs a unimanual approach whereby the unimpaired hand is constrained in a glove to encourage intensive unimanual training of the hemiplegic arm. ${ }^{5}$ There is speculation that BIM may benefit later bimanual coordination, whereas mCIMT may achieve earlier capability in the hemiplegic hand due to specificity of training. Other conclusions have been drawn from animal studies hypothesising that early mCIMT may have a deleterious effect on brain reorganisation (overlateralisation of corticospinal pathways), whereas equal training of both hands may reduce such an effect. ${ }^{4}$ To date, however, neither of these approaches have been tested or compared in a definitive randomised controlled trial (RCT) in very young infants with asymmetric brain lesions. This RCT, the Rehabilitation EArly for Congenital Hemiplegia (REACH) study, will directly compare an intensive infant friendly one-handed approach using mCIMT, called 'Baby mCIMT', to an equally intensive two-handed (bimanual) approach, called 'Baby BIM,' in very young infants with unilateral or asymmetric brain lesions and clinical signs of unilateral CP.

\section{AIMS AND HYPOTHESES \\ Broad aim}

To conduct a multisite RCT in four states, in Queensland, New South Wales, Western Australia and Victoria (Australia) with 150 infants (75 in each group) with asymmetric brain lesions (congenital or acquired prior to 6 months ca) and clinical signs of UCP. This study will determine whether Baby mCIMT is more effective than Baby BIM in improving infants' ability to use their impaired hand in bimanual play activities (Mini-Assisting Hand Assessment (Mini-AHA)) at 12 months corrected age (ca). The secondary outcomes will test the differential effect of each approach on fine motor (Hand Assessment of Infants (HAI) ) and Bayley Scale for Infant and Toddler Development (Bayley-III; motor and cognitive domains) at 12 and 24 months ca. in addition to symmetry of the corticospinal pathway development and its relationship to bimanual activities at 24 months ca.

\section{Major hypothesis to be tested}

$\mathrm{H}^{1}$ Baby mCIMT improves the symmetrical development of reach and grasp (unimanual capacity, HAI) to a greater extent than Baby BIM leading to better bimanual coordination (Mini-AHA) at 12 months ca.

\section{Secondary hypotheses}

$\mathrm{H}^{2}$ Baby mCIMT is more effective than Baby BIM in enhancing fine motor and cognitive development at 12 and 24 months ca (Bayley-III) and better participation in play, self-care activities and social functioning (Pediatric Evaluation of Disability Inventory Computer Adapted Test (PEDI-CAT)).

$\mathrm{H}^{3}$ neuroplasticity of the key motor networks, as measured by structural connectivity, is enhanced and associated with improved bimanual function at 24 months ca in infants undertaking Baby mCIMT compared with those receiving Baby BIM therapy.

The primary aim of our study is to test the major hypotheses in an RCT conducted according to Consolidated Standards of Reporting Trials (CONSORT) guidelines ${ }^{6}$ where we aim to determine if Baby mCIMT confers superior and lasting benefits on use of the impaired hand in bimanual activities over Baby BIM therapy.

Our secondary aim is to determine if there is a differential impact of Baby mCIMT and Baby BIM on early cognitive and fine motor development (Bayley-III) and better participation in play, self-care activities and social functioning (PEDI-CAT) in association with early brain reorganisation (diffusion MRI (dMRI) at 24 months ca). Enhancing our understanding of the neural mechanisms underlying early development of upper limb function and bimanual coordination according to the type of intervention will provide important evidence as to the mechanisms underlying children's responses to the type of intervention and the impact on early brain development. To achieve this, we will use dMRI and structural connectivity analyses to evaluate white matter development in motor networks controlling movement in infants receiving Baby mCIMT or Baby BIM therapy at 24 months.

This clinical trial will be conducted across four states of Australia where there is access to populations of infants with hemiplegia through respective state-wide rehabilitation services and the Australian Cerebral Palsy Register (ACPR). ${ }^{7}$ Children with hemiplegia usually attend regular schools; however, severity of their hemiplegic arm impairment reduces independence in activities of daily living, compromises participation in education and leisure and can influence their later vocational aspirations. Very early rehabilitation with the most effective therapy that leads to sustained improvements in function and neurological structure could have profound effects on later participation offering a cost-effective and timely model of care. Improving our understanding of the mechanisms of response to therapy is essential to providing effective and timely treatment to achieve sustained outcomes.

\section{Evidence for $\mathrm{mCIMT}$ and bimanual upper limb rehabilitation in congenital hemiplegia}

Our meta-analysis reported the efficacy of interventions on improving upper limb dysfunction for school-aged children with hemiplegia. ${ }^{3}$ There was moderate to strong evidence that the original approach to CIMT (with a long cast on the unimpaired arm combined with intensive shaping) or modified CIMT (using a glove on the unimpaired hand with activity-based training) was more effective than usual care to improve the quality and efficiency of arm movement. When mCIMT was compared with an equal dose of bimanual therapy at school age, there were differential effects related to the specificity of training. ${ }^{8}$ It remains unclear, however, whether one approach is superior in infants as the majority of studies did not include children under 1 year of age. Despite the philosophical preference for very early intervention to optimise brain 


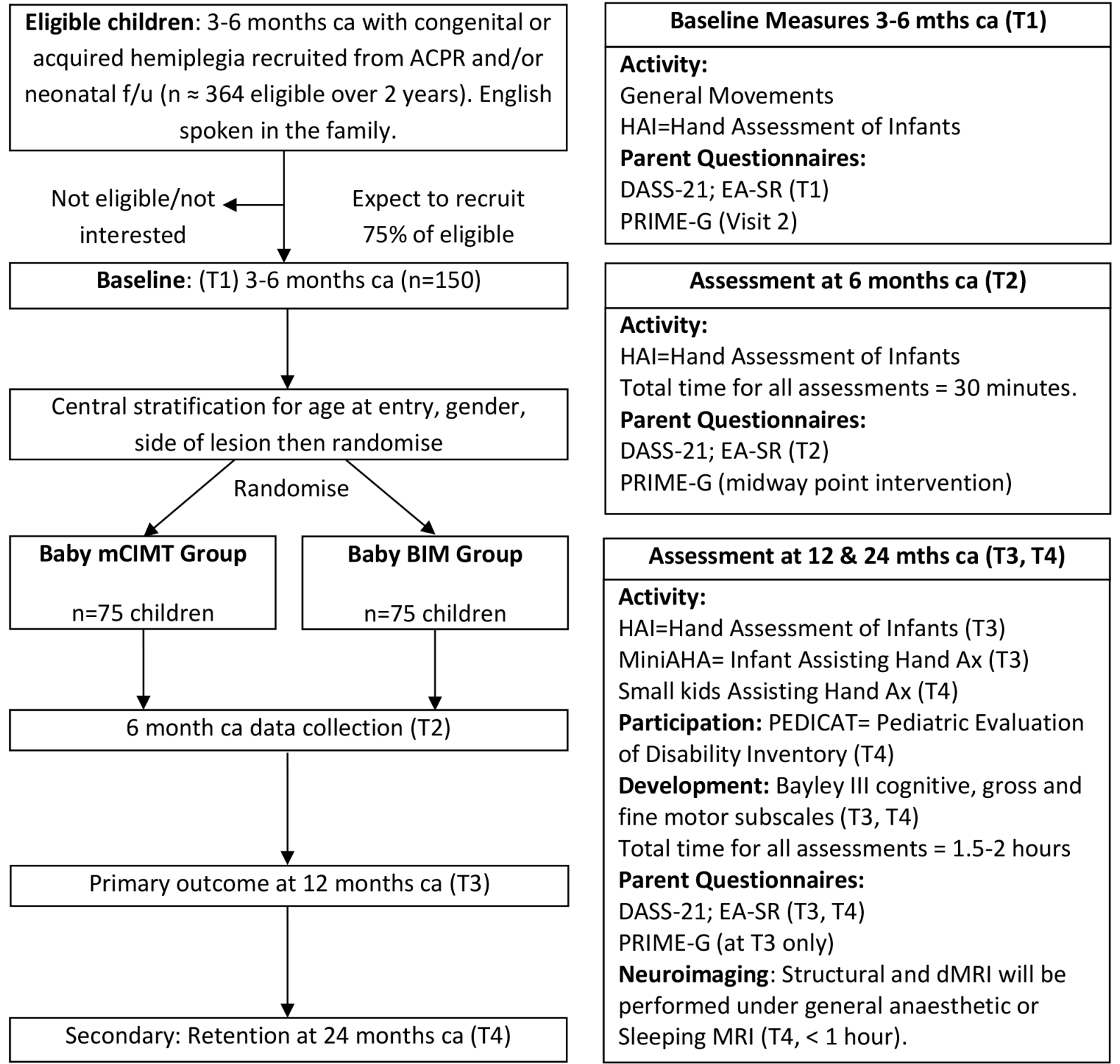

Figure 1 CONSORT flowchart. ACPR, Australian Cerebral Palsy Register; BIM, bimanual approach; CONSORT, Consolidated Standards of Reporting Trials; DASS, Depression Anxiety Stress Scale; mCIMT, modified constraint-induced movement therapy; EA-SR, Emotional Availability Self-Report Scale; PRIME-G, Pediatric Rehabilitation Intervention Measure of Engagement General Research Version.

neuroplasticity, there is limited research on early upper limb rehabilitation for infants less than 12 months ca. ${ }^{9}$ It is important to test the efficacy of these two approaches to upper limb therapy in young infants where there is greater potential to harness neuroplasticity.

Cortical reorganisation after an early brain lesion: a critical window For infants with early brain lesions, there are important phases of sensorimotor reorganisation in the first year ${ }^{10}$ with two main types of brain reorganisation. ${ }^{11}$ Ipsilesional reorganisation (ie, reorganisation within spared cortical tissue of the damaged hemisphere) allows the damaged motor cortex to become reconnected to the spinal cord, as observed in adults following stroke. ${ }^{12}$ Contralesional reorganisation (ie, reorganisation in the undamaged cortex) occurs when existing ipsilateral motor projections remain intact instead of becoming retracted within the first months of life. This alternate type of reorganisation occurs in very early brain lesions ${ }^{11}$ and can lead to severe motor impairment due to dissociation of the primary sensory and motor pathways ${ }^{13} 14$ (figure 1 . It is proposed that the first 3-6 months following an asymmetric brain lesion provides a critical opportunity for interventions to influence lateralisation of the corticospinal (CS) 
pathways. ${ }^{15}$ When sparing of the CS tract is present, early intervention may shape cortical reorganisation and improve outcomes.

To date, there is limited human evidence to determine which interventions lead to ipsilesional rather than contralesional reorganisation. Animal studies provide strong evidence that the type of training (mCIMT or BIM) may differentially impact early brain (re) organisation in infants with asymmetric brain lesions. ${ }^{16}$ A review of feline model studies supports the initiation of prehensile training in infants before 6 months. ${ }^{17}$ This review indicated a strong correlation between activation of the CS tracts and strength of synaptic connections with spinal motor circuits supporting the hypothesis that early brain damage might initiate a vicious cycle where damaged CS tracts are competitively disadvantaged from maintaining spinal synapses, resulting in secondary reductions in these connections. ${ }^{17}$ More recently, the same research group examined whether targeted activation of spared CS tracts led to functional improvement by exploring the effects of early intervention in cats with M1 inactivation. ${ }^{16}$ Three groups were studied: (1) early restraint alone with no training of the impaired limb which equals infant age forced use; (2) early restraint plus training which equals infant CIMT and (3) late restraint plus training, which equals restraint and training at feline adolescence. Outcomes measured were: (1) CS tract connections, (2) M1 motor maps and (3) motor performance. Restraint alone was able to restore CS tract connectivity yet failed to impact M1 motor maps or motor function. Late training impacted both CS tract connectivity and motor maps but failed to induce significant functional recovery. The only intervention resulting in major improvements in all three measures of outcome was early restraint combined with training. ${ }^{16}$ These animal data support infant mCIMT for rehabilitation of children with asymmetric brain lesions.

\section{Evidence from advanced brain imaging in children with congenital} hemiplegia

The importance of a multilevel network in the reorganisation of the CS system has been suggested by our work in children with congenital hemiplegia. ${ }^{18}$ The developing connectivity and symmetry of the thalamocortical pathways connecting M1 with the motor thalamus is as important as the symmetry of the CS tracts for unimanual capacity and bimanual coordination. ${ }^{18} 19$ High Angular Diffusion Imaging was performed to evaluate symmetry in the CS (motor) and thalamocortical (sensorimotor) tracts. Surprisingly, microstructural measures of the sensorimotor thalamic tracts were more significantly correlated with paretic hand function than those of the CS tracts. These data suggest functional outcome is not only related to the integrity of the CS tract but also requires feedback from sensory systems to shape the motor cortex and underlying pathways. ${ }^{15} 18$

To date, upper limb rehabilitation studies have focused on interventions to promote activation of the motor tracts alone with little regard to a balance of input to and from the sensory tracts. ${ }^{3} 18$ These data on rehabilitation and (re)organisation in UCP support a two-handed or bimanual approach to early rehabilitation. As evidence supports alternative approaches, mCIMT from animal studies and BIM from advanced MRI, in addition to the marked lack of evidence for an effective treatment for infants at risk of hemiplegia, ${ }^{20}$ there is an urgent need for further randomised clinical trials. The study proposes a direct comparison between the two alternative approaches (Baby mCIMT and Baby BIM) in very young infants at risk of hemiplegia.

\section{METHODS}

Full ethical approvals for this study have been obtained from the relevant sites in Queensland, Perth, Melbourne and New South Wales. Written and informed consent will be obtained from all parents or guardians of participants before entering the trial. This trial has been registered with the Australian New Zealand Clinical Trials Registry (ACTRN12615000180516) ${ }^{21}$ This study protocol is reported according to the Standard Protocol Items: Recommendations for Intervention Trials statement (SPIRIT; 2013).

\section{Study design}

A single-blinded randomised comparison trial will compare Baby mCIMT and Baby BIM in infants with congenital or early acquired hemiplegia with unilateral or asymmetric brain lesions according to CONSORT guidelines (see figure 1).

\section{Recruitment}

Participants

One hundred and fifty infants aged between 3 and 6 months ca at study entry with unilateral or asymmetric brain lesions (identified on cranial ultrasound or MRI) prior to 12 weeks post-term and clinical signs of hemiplegia will be recruited. These infants will be identified from neonatal follow-up clinics at the following sites in four states of Australia: in Queensland at The Lady Cilento Children's Hospital (LCCH), Royal Brisbane and Women's Hospital, Mater Mothers Hospital, Sunshine Coast University Hospital and the Gold Coast University Hospital; in New South Wales at The Cerebral Palsy Alliance and the Children's Hospital at Westmead, Sydney; in Victoria at The Royal Children's Hospital and Monash Medical Centre Melbourne and in Western Australia at The Perth Children's Hospital and the King Edward Memorial Hospital for Women, Perth, in addition to the ACPR. It is predicted that 364 infants will be born with asymmetric brain injuries over the 2 years of recruitment, so that recruitment of 150 infants ( $41 \%$ of eligible) is feasible.

\section{Inclusion criteria}

Participants will be recruited at $\leq 6$ months ca ( +14 days), have English spoken in the family and have the following by 6 months (+14 days) ca: 
1. Asymmetric brain lesion identified on cranial ultrasound or MRI including asymmetric (one-sided or more involved on one side) or unilateral brain injury including:

- Preterm or term arterial stroke;

- Grade III or IV intraventricular haemorrhage;

- Asymmetric periventricular leucomalacia;

- Asymmetric deep grey matter lesions; and

2(a): Absent Fidgety Movements on General Movements Assessment (GMs) at 12 weeks ca; or

2(b): Abnormal Hammersmith Infant Neurological Examination (HINE) between 18 to 26 weeks ca; the Hammersmith Infant Neurological Evaluation (HINE) is a standardised neurological examination of cranial nerve function, posture, quality and quantity of movement, tone and reflexes and reactions, which has numeric scoring. A HINE score at 3 months $<57$ is $96 \%$ predictive of cerebral palsy. ${ }^{22-25}$

and

3. Asymmetry of upper limb reach and/or grasp on the HAI (>3 point difference) that is congruent with the asymmetry found on the early cranial ultrasound (CUS) or brain MRI (ie, opposite to likely side of the lesion).

\section{Exclusion criteria}

Infants will be excluded if they have:

1. Epilepsy uncontrolled by medication as this would be a confounder;

2. Retinopathy of prematurity $>$ grade two or cortical blindness whereby visual tracking on clinical assessment is not possible will be excluded;

3. Ventriculo-peritoneal shunts.

\section{Confirmation of diagnosis/motor distribution}

At 12 months ca, the diagnosis of congenital hemiplegia or UCP will be confirmed by a paediatrician, rehabilitation physician or neurologist using a structured proforma of motor type and distribution. HINE will be performed at 12 and 24 months ca.

Brain MRI at 24 months ca will assess asymmetry of corticospinal and thalamocortical tracts and unilateral/ bilateral brain lesions using both a semiquantitative scale $^{26}$ and quantitative analysis of microstructure on dMRI, which will enable secondary subgroup analysis (unilateral/asymmetric bilateral).

\section{Comorbidities}

Presence and severity of epilepsy (controlled by medication), visual fields (hemianopia) and/or hearing deficits will be assessed by a medical physician at 12 months ca.

\section{Randomisation process}

Children will be randomised centrally at the Queensland site to receive either Baby mCIMT or Baby BIM using a concealed centralised electronic allocation system determined by non-study personnel. After baseline assessment and informed consent, children will be stratified centrally for age at entry (3-4/5-6 months), gender (male/female), side of lesion (right/left) then randomised. Allocation will be confirmed to the local study therapist from the central study coordinator after the participant has been enrolled and baseline data have been collected.

\section{Blinding}

Structural MRI data will be qualitatively and quantitatively analysed at 24 months ca by a child neurologist masked to group allocation and previous history. Data for the Mini-AHA and Small-kids AHA at 12 and 24 months ca, respectively, will be rated from videos by an independent accredited rater masked to group allocation. Analyses will be performed using coded study allocation.

\section{Study procedure}

\section{Study interventions}

i. Infant-friendly modified constraint-induced movement therapy (Baby mCIMT): comprises restriction of use of the unimpaired limb using a simple restraint such as a glove, sock or sleeve with a bag clip combined with intensive play-based activity with the hemiplegic arm (table 1).

Theoretical framework of Baby mCIMT: Baby mCI$\mathrm{MT}^{27}$ is a further modification of mCIMT, ${ }^{28}$ adapted to very young babies at high risk of developing unilateral CP. In the present clinical trial, Baby mCIMT has been designed in close collaboration with Professor Ann-Christian Eliasson who developed Baby CIMT and eco-CIMT. ${ }^{29}$ Baby mCIMT aims to improve the ability of the 'impaired' hand at a very early age, when movement skills are developing and when neural plasticity may allow interventions to be more effective. ${ }^{17}$ To achieve this, some 'restraint' of the non-impaired hand is typically needed during the practice time. Children are stimulated to use the impaired hand through presentation of a carefully selected set of age appropriate and engaging toys and play objects. Practice will be implemented in infants' daily environments by their parents under the guidance of study therapists.

Baby mCIMT considers both developmental and ability constraints as well as perceptual, cognitive and environmental influences on the use of the impaired arm/hand. Physical assistance will be kept to a minimum but may be used judiciously to facilitate Baby mCIMT. Toys/tasks will be carefully selected for their developmental, therapeutic and engaging properties, and the environment may be manipulated to support the child with achieving unimanual skill development. Baby mCIMT is approached from a number of different perspectives:

- For children with UCP, the ability to use their involved hand for reaching, grasping and object manipulation does not develop at the same rate or in the same way as that of their non-involved (preferred) hand. The impact of unilateral impairment on hand use needs to be considered to organise the intervention both from a 


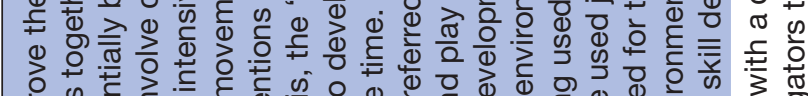

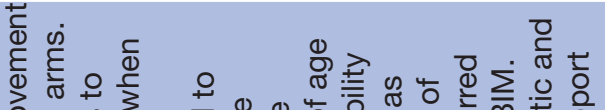

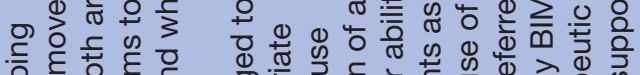

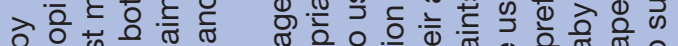

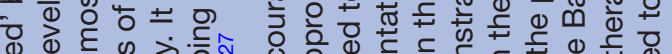
$\Phi$ d क ᄃ

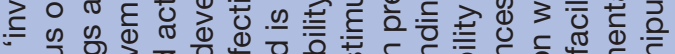

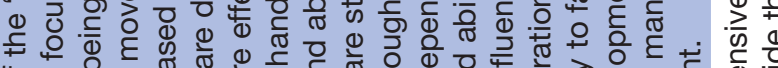

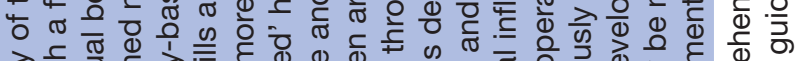

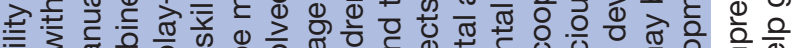

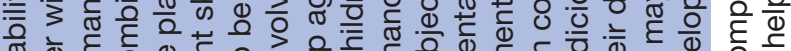
ब ब

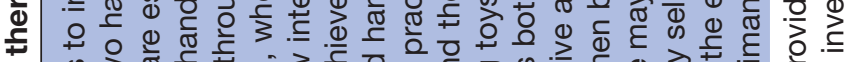
ब

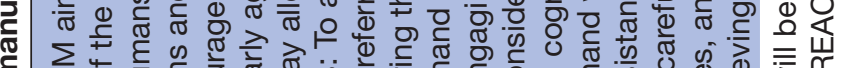
ऐ娄 万人 两 क人
है $\frac{0}{0}$

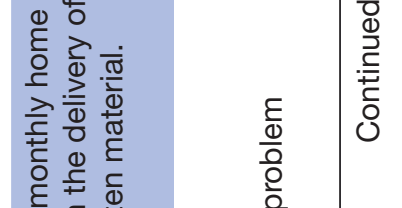

劫要弯

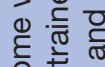

药

त船

$\sum \frac{\pi}{0} \frac{0}{0}$

$\Phi$ 艺 $\frac{\Phi}{\Phi}$

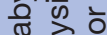

ॠ

亏े

तथ

कृ 중

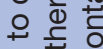

万人

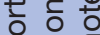

윽 융

क गे

هั.

亨

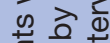

के

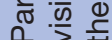

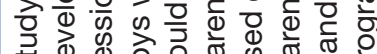
का

ช 0

in 요

을

응

至. 薁

5 인

Ф)

을 흔 긍

放 $\frac{0}{0}$

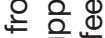

Dी की के

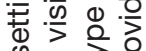

क 仓 क

品 오 중

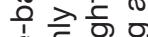

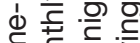

우 을 뉜 흥
茫

$\stackrel{1}{7}$

$\stackrel{\vec{F}}{\stackrel{P}{\circ}}$

음

음

$\frac{\pi}{2}$

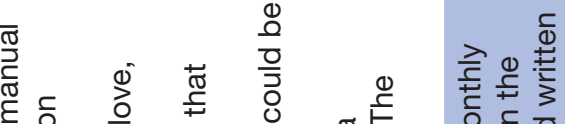

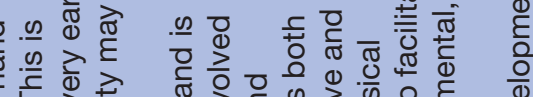

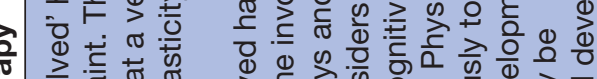

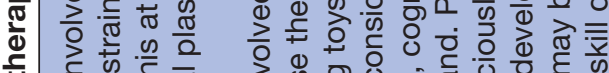

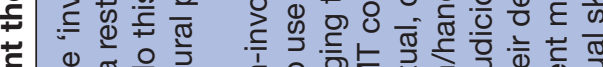

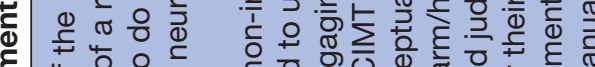

छั⿻

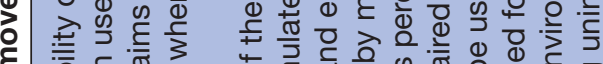

券

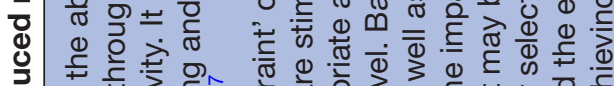

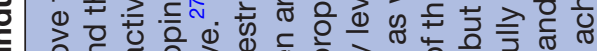

ᄃ

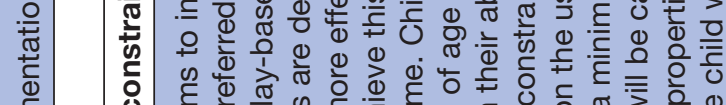

है

更 ब

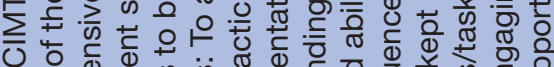

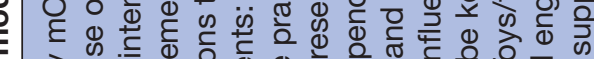

음

纹 즣

苛

ᄃ خ

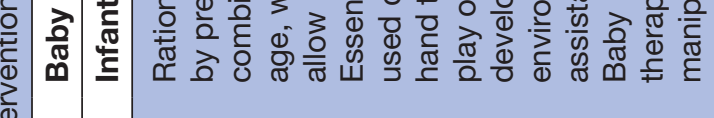

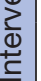

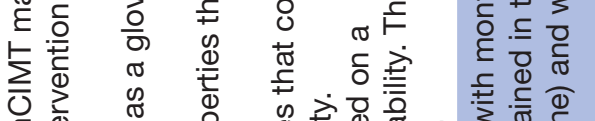

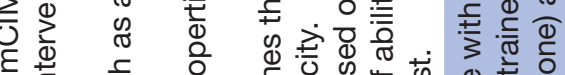

ป.

उ.

西

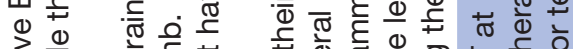

के

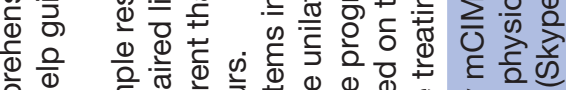

है 등

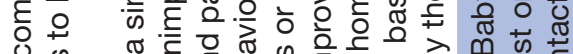

u क व

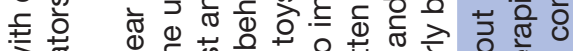

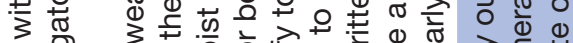

व

ब

궁 क을

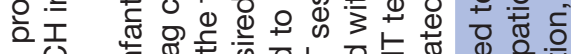

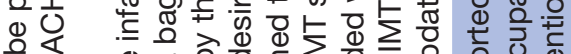

넝 ब

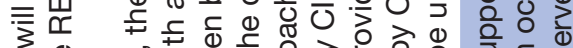

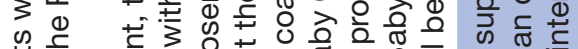

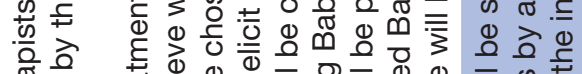

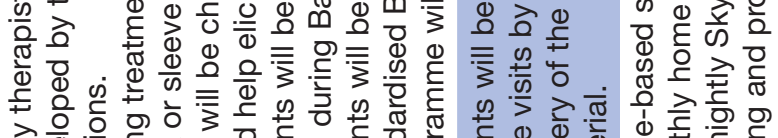

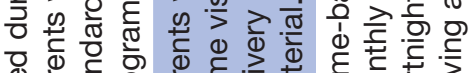

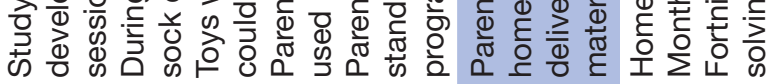

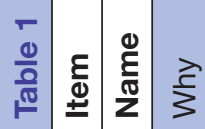

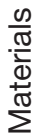

$\stackrel{ }{\frac{?}{3}}$

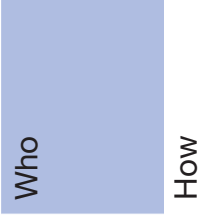


developmental perspective and also to target the correct motor ability level.

- Children's interests, motivation, cognitive and perceptual capacities, will be important considerations when selecting toys/tasks to promote use of the impaired hand/arm.

- Parents will be supported to carry out Baby mCIMT at home with monthly home visits by an occupational therapist or physiotherapist trained in the implementation of the intervention, remote contact (Skype/Facetime/WhatsApp or telephone) between home visits and written material.

ii. Baby BIM: comprises play-based activity designed to encourage the use of both the impaired and unimpaired upper limbs during bimanual activities. Theoretical framework of Baby BIM: In contrast to Baby mCIMT, Baby BIM uses an approach that encourages the two hands to be used together with a focus on developing bimanual skills. Bilateral arm movements are the predominant pattern of upper extremity movements in the first year of life,${ }^{30}$ as humans are essentially bimanual beings. The developmental sequence of bimanual skills observed during infancy are related to the infant's postural, sensory, perceptual and cognitive development as well as unilateral hand skill development. ${ }^{31}$ It is currently not known whether improvements in unilateral hand skill development will automatically lead to improvement in bimanual performance or whether focussing on developing specific bimanual skills would be more effective.

Similar to Baby mCIMT, Baby-BIM is designed for very young children up to approximately 12 months ca who have or who are 'at risk' of developing UCP. Baby BIM aims to improve the ability of the involved hand at a very early age, when movement skills are developing and when neural plasticity may allow interventions to be more effective. ${ }^{17}$

Baby BIM considers both developmental and ability constraints as well as perceptual, cognitive and environmental influences that may impact the use of two hands. Physical assistance may be used judiciously to encourage motor activity. Toys and tasks are carefully selected for their developmental, therapeutic and engaging properties, and the environment may be manipulated to support the child undertake bimanual activity.

Baby BIM is approached from a number of different perspectives:

- An understanding of the bimanual motor actions which exist at different ages is required.

- For children with UCP, the ability to use their involved hand for reaching, grasping and object manipulation does not develop at the same rate or in the same way as that of their uninvolved hand. The impact of the unilateral impairment on bimanual hand use needs to be considered to organise the intervention both from a 
developmental perspective and also to target the correct motor ability level.

- Children's interests, motivation, cognitive and perceptual capacities, which develop over this first year of life, are important considerations when selecting toys/tasks to promote bimanual skills.

- Parents are supported to carry out Baby BIM at home with monthly home visits by an occupational therapist or physiotherapist trained in the delivery of the intervention, remote contact (Skype or telephone) and written material.

\section{Implementation of Baby mCIMT and Baby BIM}

Preparation and planning of intervention sessions will be an important part of implementing both the Baby mCIMT and Baby BIM programmes. Study therapists will be provided with comprehensive Baby mCIMT and Baby BIM manuals developed by the REACH investigators to help guide their intervention sessions. The manuals will include important background knowledge about the typical sequence of upper limb development from zero to 12 months. Three ability levels have been developed for the Baby mCIMT and Baby BIM programmes to guide intervention implementation based on ability levels observed when completing the HAI. ${ }^{32}$ These are:

Level 1: Reaching and early grasping

Level 2: Grasping

Level 3: Refinement of grasp and object manipulation

Although these levels are age related in typically developing infants, the motor, cognitive and developmental level of each child with or at risk of UCP needs to be the primary consideration in identifying the appropriate level at which to target Baby mCIMT or Baby BIM. For each of the three ability levels, comprehensive information is provided in the intervention manuals to guide therapy. This includes: (1) an overview of development of unimanual or bimanual ability at this level, (2) an overview of perceptual and cognitive influences, (3) principles of intervention at that level for each model and (4) a table providing specific treatment strategies organised by person, environmental and task considerations for each skill.

Using these levels aims to facilitate selection of important actions to practice at the right ability level to support the development of unimanual and/or bimanual skills. ${ }^{33}$

Use of assessment information for treatment planning

Information from completing the HAI and clinical observations of posture and movement will be used to identify the level at which unilateral and bimanual play activities should be targeted. ${ }^{27}$

- Young babies (3-4 months of age) and babies whose HAI result shows they may not have the ability to grasp with the involved upper limb will commence at level 1.
- Babies whose HAI result shows they have rudimentary grasping skills with the involved upper limb will start at level 2.

- Babies whose HAI result shows that they have good grasping skills in the involved upper limb and require further skill development will start at level 3.

- Over the course of the intervention period, as the infant develops new hand skills, they will be progressed through the levels of ability to ensure the continued 'just right challenge' and to continue to refine and improve the child's hand and arm ability.

Venue: home programme is optimal for best practice

The most effective partnership-based home programmes include five steps: (1) establishing a collaborative partnership, where the parent is the expert in knowing their child and their home environment; (2) having the child and family (not the therapist) set goals about what they would like to work on in the home environment; (3) establishing the home programme by choosing evidence-based interventions that match the child and family goals and empowering the parents to devise or exchange the activities to match the child's preferences and the unique family routine; (4) providing regular support and coaching to the family to identify the child's improvements and adjust the complexity of the programme as needed and (5) evaluating the outcomes collaborative. ${ }^{34}$ This study will adopt these evidencebased principles in the development of the home programme.

\section{Intensity of intervention}

The REACH trial requires parents to complete play sessions to provide a specific dose of intervention per day, with the same dose in both Baby mCIMT and Baby BIM models of intervention. The proposed optimal dosage varies according to age, as follows:

- Infants aged 3-6 months ca: 20 min per day, 5 days per week;

- Infants aged 6-9 months ca: 30 min per day, 5 days per week;

- Infants aged 9-12 months ca: 40 min per day, 5 days per week.

The dosage does not need to be completed in one session. Play sessions may be spread over the day. For example, a child of 10 months may have four sessions of $10 \mathrm{~min}$ to achieve $40 \mathrm{~min}$ of intervention per day. To date, there are no evidence to support the optimal dose for intervention according to the child's age. The session times proposed in the REACH trials are shorter than those used with older children because: (1) babies' attention spans are short and (2) small doses allow time for families to attend to the multiple demands on their time and to fit intervention between other needs such as feeding, physical care and sleeping. The total overall dose will be 70 to 89.2 hours by 12 months ca depending on infant's age at the time of recruitment (between 3 and 6 months ca). 
Procedures for both interventions

Baby mCIMT and Baby BIM will be implemented by parents in the home environment, supported by an occupational therapist or physiotherapist trained in the delivery of each intervention. Home visits will be conducted once per month from the time of recruitment until 12 months ca. At the initial home visit baseline assessments will be completed following which infants will be randomised to one of the two interventions. A further aim of the initial home visit is to understand the family environment and determine the learning and support needs of the family, provide education about the intervention to which the infant has been allocated and establish a home programme. Subsequent home visits will occur once per month and are expected to range between 1 to 1.5 hours duration. During the home visit, therapists will collect information since the previous visit or remote contact with the family, review the home practice diary and home programme, complete a play session in collaboration with the family and plan and document the next focus areas for intervention. Two weeks after each home visit, the therapist will contact the family via Skype or telephone, depending on the preferences of the family. The aim of this contact is to support the family in the delivery of the home programme, review the infant's progress and update the home programme as required.

Both programmes have: (1) the same dosage, (2) varied construct and (3) are delivered by parents in the home environment with the support of an occupational therapist or physiotherapist. To ensure the same treatment dose is delivered within and between each treatment arm, a range of strategies will be implemented. The intervention manuals developed for Baby mCIMT and Baby BIM clearly describe the treatment dose and scheduling. Computerised scheduling will be established in a Research Electronic Data Capture (REDCap) database, which will provide therapists with a prompt for intervention contacts. At the completion of each home visit and remote contact, the therapist will complete an online questionnaire (Qualtrics) which will record any deviations from the dose protocol and reasons and the amount of contact time provided. The questionnaire will further prompt therapists about the scheduling for the next contact with the participant. In planning for any potential setbacks, more than one therapist at each site will be trained to deliver each intervention. In the case of a missed home visit or remote contact, therapists will reschedule the appointment within a 2-week time frame. Each family will complete a home practice diary, which will be reviewed by the therapist at each home visit and uploaded on a central database (REDCap). The home practice diary will record the amount of daily home practice (in minutes), the activities practised and any questions for the therapist at the subsequent contact.

\section{Role of the study therapist}

Consistent with the principles of shared decision-making, ${ }^{35}$ the therapist will work in partnership with the family to determine how and when intervention will be undertaken so as to accommodate daily family routines while adhering to intervention protocol. Ensuring that the parent feels emotionally supported, the therapist will provide the rationale for the specific intervention being undertaken. Drawing on the parent's knowledge of the child together they will tailor the activities relevant to either Baby mCIMT or Baby BIM. Through information exchange, the therapist will situate the solutions for how to progress the intervention with the family thereby reinforcing a sense of self efficacy in the delivery of the intervention. ${ }^{36}$ Along with adopting a solution-focused coaching approach ${ }^{37}$ during home visits, the therapist will remain in regular telecommunications contact to help resolve any implementation challenges as these may arise.

\section{Positioning the infant for the therapy programme}

During study intervention, the infant should be in as upright and stable a position as possible. This allows the infant to more easily use goal-directed arm movements with their attention focused on the play situation. ${ }^{38}$ At times, in the HAI first ability level, play in semisupine nestled in the parent's lap and facing the parent may be used to orient infants towards toys and other play objects. The prone position should be avoided as this is a difficult position in which to encourage unimanual or bimanual skills. A baby chair/high chair is preferable for the programme once an infant has head control in supported sitting and when the baby only needs to be held around the trunk when sitting on a lap. The infant should be stable when using an infant seat, and appropriate supports may be required to improve stability. The child should not be practising sitting balance when the focus is on use of upper limbs and hands as the quality of postural control is known to influence exploration and the quality of object manipulation. ${ }^{39}{ }^{40} \mathrm{~A}$ table at the appropriate height and close to the body is also encouraged as it is useful for eliciting grasp of toys placed in various positions.

Supporting the parent-infant relationship and parent mental health Both interventions will be conducted in a manner consistent with supporting parental mental health and sensitive, responsive parenting. Dr Koa Whittingham, a registered clinical and developmental psychologist and investigator on this project, is responsible for developing the parental mental health and parenting support component of the intervention manuals, for training all of the therapists in parental mental health and parenting support and for providing ongoing mentorship and advice to therapists as needed throughout the trial. The parental mental health and parenting support components will be developed grounded in three key theoretical frames: the emotional availability literature, ${ }^{41}$ the grief and loss literature including chronic sorrow theory ${ }^{42}$ and Acceptance and Commitment. ${ }^{43}$ Training will focus on enabling therapists to better support parental grief and to recognise and support responsive parenting. If parental mental 
health or the parent-infant relationship is found to be a significant concern, either arising directly from therapist-parent contact or from parental scores on the Depression Anxiety Stress Scale (DASS) or Emotional Availability Self-Report Scale (EA-SR), then appropriate referral options will be discussed with the parent.

\section{Delivery and fidelity}

The United States National Institute of Health Behaviour Change Consortium has recommended a model of fidelity comprising five areas for consideration. ${ }^{44}$ The five areas encompass study design, training of intervention providers, treatment delivery, treatment receipt and enactment. While this model was developed for psychological interventions, it has been adopted in rehabilitation focused research. ${ }^{45} 46$ The model provides an overarching framework for addressing fidelity in the current study. All therapists implementing Baby mCIMT and Baby BIM will participate in standardised training. Training will include certification in conduct of the HAI (2 days), Mini-AHA if not already certified (3 days) and intervention delivery and study procedures (2 days). The standardised training for intervention delivery and study procedures will be video recorded to be used if new study therapists require training over the 4-year study period. Standardised training manuals will be provided for each study therapist. The standardised training will include both didactic and interactive elements encompassing the rationale underpinning each intervention, engaging and working effectively with families, principles of family-centred practice and coaching, infant ability levels determined through the HAI, intervention planning, toy selection, implementation of Baby mCIMT and Baby BIM including video case vignettes. In addition, therapists will be familiarised with the intervention manuals and a series of nine worksheets that will be used in the implementation of each intervention protocol to further enhance standardisation of intervention delivery. Four of these worksheets are for the study therapists to prompt the delivery of essential elements of the intervention. These include a mind map to guide therapists in provision of necessary information about each intervention to the parent, a guide and checklist for structuring home visit sessions, a standardised home programme template and questionnaire completed by the study therapist at the conclusion of each home visit to reflect on their performance and engagement with the family.

A questionnaire using a five-point Likert scale developed for the study will evaluate the therapists' self-perceived knowledge, skills, beliefs and confidence in delivering each intervention. This will be administered prior to and at the completion of the standardised training, and then at $6,12,18$ and 24 months post to monitor any potential drift and implement booster sessions if indicated. Monthly Skype/web-based meetings among study therapists will occur to provide ongoing mentorship and support to implement the interventions. Each study therapist will have a chief investigator at their local site to provide support, supervision and mentorship.

A provider delivery observational checklist will be developed to evaluate treatment adherence, treatment differentiation and therapist competence in delivery of core intervention components. Essential and proscribed elements of each intervention will be defined based on the intervention manuals and training resources. Core elements will be worded into observable actions and behaviours to reflect both adherence to the protocol and the quality by which the interventions have been delivered (competence items). A coding manual will be developed for each item on the checklist. Content validity of the checklist will be determined by an expert panel comprising REACH study investigators and developers of the intervention manuals and standardised training. To determine if the items measure what they intend (ie, adherence, competence, treatment differentiation), an expert panel comprising REACH study investigators will review each item in the checklist. Inter-rater and intrarater reliability will be established.

Study therapists will videotape all home visit sessions. Videotaped sessions will be uploaded centrally (Queensland site). The first two home visits for each therapist delivering each intervention will be reviewed independently by two study investigators at the Queensland site against the Provider Delivery Observational Checklist. A random sample of videos will then be selected over the course of the study so that approximately $10 \%$ of each therapists' intervention sessions will be evaluated using the Provider Delivery Observational Checklist. A threshold of $80 \%$ compliance to checklist items will be applied and deviations in excess of this will result in feedback to the study therapist's local site supervisor. The supervisor and study therapist will then review and determine strategies to mitigate further deviations from protocol.

A range of strategies will be employed by the study therapists for monitoring and improving the receipt of treatment. Worksheets developed to enhance the parents' delivery of the intervention include a guide to preparing for a play session, choice of restraint (for Baby mCIMT group only), a mind map (Are you ready to play?) to prompt parents around the optimal time and environment to play with their child and a mind map (How did the session go?) to encourage parents to reflect on their play session with their child. As part of the structured home visit, study therapists will ask the parents to demonstrate the intervention activities they have been practising with their child and the toys they have selected. Therapists will provide feedback on parent performance and highlight the child's progress. As the child progresses in development of their hand skills, therapists will provide demonstration of the next stage/goals of therapy and discuss features of objects/toys that may elicit the behaviours that are being targeted. Therapists will employ coaching strategies with the families to help them consider toys/ objects they could use in their own environment. Collaborative discussions will enable therapists to determine if 
the parents understand the next therapy goals. Families may videotape their child during the session as a prompt to assist them in the delivery of the treatment in between therapist contacts.

Parents will be independently interviewed when their infant at baseline, midway through each infant's intervention period and at 12 months ca to determine how they are reacting to the intervention requirements, the extent to which delivery is feasible in their family context and their reaction to changes they may have observed in their child. This interview will follow parent's completion of the Pediatric Rehabilitation Intervention Measure of Engagement (General Research Version) (PRIMEG). The PRIME-G has been developed to gain parent's perspective on their own engagement in their children's therapy.

\section{Intervention content and implementation}

Details of the implementation of Baby CIMT and Baby BIM are presented according to the

template for intervention description and replication (TIDieR) checklist and guide in Table $1{ }^{47}$

\section{Concurrent therapies}

Concomitant interventions (eg, PT, OT) provided during the study period until 24 months ca will be recorded using a logbook. Infants are not expected to receive intramuscular botulinum toxin A to the upper limb as it has not received regulatory approval for infants with CP less than 2 years of age in Australia. Rigid casting will be discouraged as there is no published evidence of functional benefits for infants with hemiplegia less than 24 months ca. Night splinting, if used will be recorded in the logbook.

\section{Outcome classification and exit criteria}

Infants recruited to the study will be assessed at specific time points including at 6 months ca for cross-sectional analysis of the study population on asymmetry of reach/grasp (HAI), at the conclusion of intervention at 12 months ca on bimanual coordination (Mini-AHA) and general development (Bayley-III). The diagnosis of hemiplegia (by motor type and distribution) will be confirmed independently by medical personnel at 12 months ca using a structured proforma. If there is deterioration of development of hand skills of the less impaired hand in either intervention group quantified as a difference of $<3$ points on the unimpaired hand compared with the impaired hand, the case will be presented to the independent data monitoring and safety committee (DMSC) to determine if stopping rules have been met.

\section{Adverse events}

Any adverse events associated with either intervention will be screened at 6, 12 and 24 months using open-ended questions asked by non-treating personnel. Families are monitored for parent mental health and parent/child attachment at baseline, 6 and 12 months ca using DASS ${ }^{48}$ and EA-SR. ${ }^{49}$

\section{Data Management Safety Committee}

Three independent researchers will comprise the DMSC. They will review all adverse events, subject participant retention in each arm of the study and compliance with study protocol at 12 monthly intervals.

\section{Baseline assessments at study entry}

Measurement of unimanual capacity will be performed using the $\mathrm{HAI}^{27}{ }^{32}$ at study entry, then at 6 months ca to describe the study sample.

1. Classification of the Early Brain Injury: The Kidokoro Scale $^{50}$ : scoring of structural MRI to classify brain injury and growth has been validated for use at term equivalent age (TEA) in infants born preterm. ${ }^{50} 51$ Qualitative scoring systems of MRI at TEA include quantitative biometrics to measure the impact of secondary brain maturation and growth following preterm brain injury. ${ }^{52-54}$ These neonatal MRI scoring systems underwent further development to include evaluation of deep grey matter structures and the cerebellum ${ }^{20}$ and this version will be used in this study for infants who underwent neonatal MRI.

2. Prechtl's General Movements Assessment (GMs): The GMs is a diagnostic tool for neurological evaluation of at-risk infants. ${ }^{556}$ It has high predictive value for neurodevelopmental outcome at 12-24 months (eg, sensitivity $\geq 92 \%$ and specificity $\geq 82 \%, p<0.01$ ). Infants will be video recorded for 5 min at 12-14 weeks ca (fidgety period) to determine 'absent fidgety' GMs as one of the inclusion criteria to the study. Videos will be recorded globally for $5 \mathrm{~min}$, then $1 \mathrm{~min}$ to focus on each hand to detect hemiplegia. ${ }^{57}$ The GMs will also be rated by a second masked assessor to confirm 'Absent Fidgety' or 'Asymmetric Absent Fidgety' as markers of high risk of CP and high risk of UCP.

3. HAI:This is a criterion, norm referenced assessment, which evaluates quality and frequency of hand abilities from 3 to 12 months post-term. Test items were developed from a review of the literature, expert panel and systematic observations of infants. A Rasch measurement model was used to determine internal scale validity. The test items assess unilateral and bimanual actions, based on the frequency of occurrence and quality of performance. The HAI comprises 17 items, which describe object-oriented hand actions scored on a three-point scale. For 12 items, each hand is scored separately, which enables quantification of asymmetry between limbs. A further five items evaluate bimanual hand performance. Scores from each section are summed to provide a Both Hands sum score, which is converted to an interval level $(0-100)$ Both Hands Measure. The HAI will be performed at baseline, 6 and 12 months ca. ${ }^{58}$ Videos of the 6 and 12-month assessments will be assessed independently by a certified rater. ${ }^{59}$

4. Demographic questionnaire: The demographic questionnaire has been developed specifically for the study to assess family factors and demographic 
variables such as socioeconomic status, parental education and child factors such as birth history and other comorbidities. A measure of social advantage/ disadvantage will also be derived from postcode of residence using the Index of Relative Socio-economic Advantage/Disadvantage (2006) from the Australian Bureau of Statistics (129 Reference). Deciles will be reported on a continuum with lower scores reflecting greater socioeconomic disadvantage and higher scores reflecting socioeconomic advantage.

\section{Classification of the sample at 12 and 24 months ca}

All participants will be classified using the:

a. Manual Abilities Classification System for Infants (mini-MACS): The mini-MACS will be used to classify the infants' ability to handle objects in daily activities on one of five levels. ${ }^{60}$ The mini-MACs version of the MACs for children aged less than 4 years will be used in this study and this has strong content validity and inter-rater reliability in both parents and therapists for infants aged 12-51 months ${ }^{61}$. The Intraclass Correlation Coefficient between parents and therapists on the mini-MACs was 0.90 (95\% CI 0.79 to 0.92$)$ and for the two therapists was $0.97(95 \%$ CI 0.78 to 0.92 ). Children will be classified on miniMACs by an occupational therapist on this measure in discussion with the caregiver.

b. Gross Motor Function Classification System extended and revised version (GMFCS-ER): Classifies self-initiated movements related to sitting/ walking over five levels. ${ }^{62}$ It is based on self-initiated movements, antigravity postures and motor skills expected in a typical 5-year old. Children who are independently ambulant are classified as GMFCS I or II, those requiring an assistive mobility device to walk classified as GMFCS III and those in wheeled mobility as GMFCS IV and V. Most children in the REACH study will be GMFCS level I or II. The GMFCS has internationally established validity, reliability and stability for the classification and prediction of motor function of children with CP aged 2-12years ${ }^{63} .{ }^{64}$ It has a high inter-rater reliability $\quad$ generalisability coefficient $=0.93) .{ }^{64}$ In the current study, the $<2$ years descriptions are used. Lower inter-rater reliability is documented for the $<2$ years age band $(\kappa=0.55)$, as younger children's gross motor abilities are more variable, and less developmental information is available on which to base the classification. ${ }^{65}$ The intrarater (testretest) reliability from $<2$ to 12 years appeared to be acceptable (generalisability coefficient $=0.68$ ). The GMFCS has been correlated with a number of motor scales, as well as CP distribution and type of motor impairment. ${ }^{65}$

c. Motor Type and Distribution: Motor type of CP will be classified by a medical practitioner as spastic, dystonic, ataxic, hypotonic, choreoathetosis, mixed $\mathrm{CP}$ or unclassifiable according to Surveillance of
Cerebral Palsy in Europe guidelines. ${ }^{66}$ Distribution will classified by number of limbs impaired.

d. Classification of the brain lesion at 24 months: The nature of the brain lesion will be classified from MRIs at 24 months ca using two scales: (a) a qualitative (Krageloh Mann) ${ }^{19}$ and (b) semiquantitative classification of brain lesion severity using structural MRI (sMRI, Fiori scale). ${ }^{67}$ The American Academy of Neurology ${ }^{68}$ has concluded that a clinical brain MRI should be part of the diagnosis of CP, so that all MRIs will be conducted as part of clinical best practice. Structural MRI will be obtained using a $3 \mathrm{~T}$ scanner at one of the four sites, with at least T1-weighted high-resolution magnetisation prepared three-dimensional image, T2-weighted spin-echo image and fluid-attenuationinversion-recovery (FLAIR) sequences. Structural MRI will be evaluated by a paediatric neurologist (SF) masked to clinical features and history, for type of lesion and to describe a presumed pathogenic pattern (eg, stroke, hypoxia/anoxia, toxic, metabolic or infective). The brain lesion severity will be determined by using a semiquantitative scale (Fiori scale) that has demonstrated high inter-rater and intrarater reliability ${ }^{67}$ and strong construct validity based on dMRI and functional severity in children with unilateral CP. ${ }^{69}$ In the Fiori scoring system, raw scores for lobes, subcortical structures (basal ganglia, thalami and brainstem), corpus callosum and cerebellum are systematically calculated, where higher scores represent more severe pathology. Each hemispheric score (HS) is the sum of the lobar scores (maximum score of 12). The basal ganglia and brainstem score (BGBS) is the sum of left and right subcortical structures (basal ganglia, thalamus, brainstem and posterior limb of the internal capsule) (maximum score of 10). The global score is the sum of the right and left HS, BGBS, corpus callosum and cerebellum scores (maximum of 40). A laterality index will be calculated for each scan (range $0-1$ ) to determine the lateralisation of the lesion, assuming the HS as the lateralised measure. A score approximating 0 indicates a more bilateral lesion; a score approaching one more unilateral involvement.

\section{PRIMARY OUTCOMES}

The primary outcome measure at 12 months ca is use of the impaired hand as an assisting hand in bimanual activities on the Mini-AHA which will be rated blinded to group allocation/order from video recordings.

\section{Bimanual performance}

All infants will be assessed using the Mini-AHA at 12 months ca and Small Kids AHA at 24 months ca. Both of these tests have been developed using Rasch methodology and measure the effectiveness with which a child 
makes use of their impaired hand during bimanual activity performance. Test-retest reliability is high for school children (ICC 0.98). Small Kids AHA is responsive to change following upper limb intervention ${ }^{5}$ with a smallest detectable difference of 3.89 raw scores. The Mini-AHA was developed for children 8-18 months and comprises 20 items rated on a four-point scale. There is evidence for internal scale validity and the Mini-AHA distinguishes between levels of ability with high precision. ${ }^{70}$ The test developers have demonstrated longitudinal validity of the Small Kids AHA and predictive validity of future use of the impaired hand as an assisting hand use. ${ }^{71}$

\section{SECONDARY OUTCOMES \\ Bayley-III ${ }^{72}$}

Infants will be assessed on the Bayley-III (cognitive and motor) at 12 months and 24 months ca. Test-retest reliability is 0.67 (fine motor) and 0.83 (gross motor) ${ }^{73}$

\section{Paediatric Evaluation of Disability Inventory Computer Adapted Test (PEDI-CAT)}

The PEDI-CAT is a norm referenced, parent-reported measure of domains of self-care, mobility and social functioning which has good validity and reliability. ${ }^{74}$ The PEDI-CAT is completed online at 12 and 24 months ca and will be used to compare outcomes between groups.

\section{$E A-S R^{49}$}

EA-SR is a 32 item self-report measure of emotional availability with excellent reliability and validity.

\section{DASS- $21^{48}$}

DASS-21 is a 21-item questionnaire that assesses symptoms of depression, anxiety and stress in adults. Respondents rate items on a four-point Likert scale reflecting how much the statement applied to them in the past week. DASS produces three subscales each with good internal consistency: the depression $(\alpha=0.91)$, anxiety $(\alpha=0.84)$ and stress $(\alpha=0.90)$ scales. DASS also has good discriminant and concurrent validity. ${ }^{48}$

\section{PRIME-G}

PRIME-G is a parent report of engagement in the intervention. This measure is part of a suite of measures of engagement currently under development (http://primeresearchteam.com/). PRIME-G is a self-report measure comprising 12 items which reflect affective, behavioural and cognitive aspects of engagement. Items are scored on a seven-point Likert scale. It is anticipated that the more highly engaged the family the more likely they will be to complete the intervention protocol. PRIME-G will be assessed at the first home visit conducted by the research occupational therapist, then at the midway point of the intervention (3 to 4 months following commencement) and at 12 months of age at completion of the intervention.

\section{Neurodevelopmental Assessment}

Neurodevelopmental assessment of the infants, will be undertaken by a medical practitioner (paediatrician, rehabilitation specialist or neurologist) at 12 and 24 months of age to confirm the diagnosis, to classify the motor type and distribution using HINE. ${ }^{22-25}$ HINE is the most accurate method for early detection of cerebral palsy in infants older than 5 months (corrected for prematurity) but less than 2 years old to detect neurological dysfunction (90\% predictive of cerebral palsy). An infant with a HINE score lower than 73 (at 6, 9 or 12 months) is considered to be at high risk of cerebral palsy. A HINE score lower than 40 (at 6, 9 or 12 months) strongly indicates cerebral palsy. ${ }^{23} 24$

\section{Neuroimaging measures}

All participants undertaking MRI will be checked for 3T MRI safety including no metal implants or ventriculoperitoneal shunts. As participants will be 24 months ca at MRI, and unable to lie still in the noisy scanner environment, their families will be offered MRI under sedation as part of clinical follow-up. ${ }^{68}$ The American Academy of Neurology practice parameter has concluded that brain MRI should be part of the diagnosis of CP. This is routinely undertaken as part of best clinical practice after referral by their treating doctor.

\section{Diffusion imaging acquisition and white matter fibre tracking}

DMRI scans, suitable for structural connectivity analyses will be acquired on 3T scanners at LCCH, Brisbane, Murdoch Children's Research Institute in Melbourne and the Children's Hospital at Westmead in Sydney and the Sir Charles Gardner Hospital in Perth. The research sequence will be acquired at the same time as clinical scans at 24 months ca during natural sleep or using sedation or under general anaesthesia (determined on a case by case basis) ${ }^{68}$ where (Note: dMRI adds $10 \mathrm{~min}$ to scan time, total scan time $35 \mathrm{~min}$ ). There are some risks associated having a general anaesthetic to undertake the MRI. In ambulant children with CP with no other major medical conditions (no uncontrolled seizures) the risk associated with general anaesthesia is $<1 \%$ chance of some complication. The referring doctor will discuss this with the parents in relation to their child. Where families do not wish their child to undergo general anaesthesia for MRI at 24 months ca, they will be offered a desensitisation programme to familiarise the child to the scanner noises so that sleeping scans can be performed. Families who do not wish ${ }^{75}$ for their child to have an MRI at 24 months can participate in the study without performing the scan.

Diffusion-weighted images will be acquired along 64 directions, with full brain coverage. DMRI data will be preprocessed to reduce artefacts, ${ }^{76}$ and the fibre orientation distribution estimated taking into account crossing fibres. ${ }^{75}$ Tractography will be performed to delineate corticospinal and thalamocortical pathways.

Quantitative diffusivity indices fractional anisotropy and mean diffusivity, will be calculated for the tracts and an asymmetry index calculated. This will be performed separately for the left/right-sided hemiplegia, cortical/ subcortical regions and uni/bilateral lesions. Our 
hypothesis is that the Baby mCIMT group will show reduced asymmetry due to enhanced plasticity.

\section{Sample size determination}

Primary basis for sample size calculation is adequate power for $\mathrm{H}^{1}$ comparison between functional effects of Baby mCIMT compared with Baby BIM therapy at 12 months ca. Based on data from a study of 2 to 3-year olds, ${ }^{28}$ we propose a mean difference of 6 AHA logit points (100-Logit Scale) ${ }^{71} 77$ as the minimum difference likely to have substantial clinical impact. Data from our large RCT comparing mCIMT and BIM in school-aged children with hemiplegia yielded SD of 12.8 and 12.6 in each group. ${ }^{8}$ Assuming a pooled SD of 12.8 units, a sample size of 144 participants (72 per treatment) will have $80 \%$ power to detect a 6 -unit difference on AHA between groups (2-sided test at $\mathrm{p}<0.05)$. We require 150 children (75 in each group) to allow for attrition (41\% recruitment of eligible is required as a minimum, therefore feasible).

\section{Statistical analysis}

Analyses will follow standard principles for RCTs, with interest focussing on comparisons between the intervention groups. Analyses will be undertaken on an intention-to-treat basis, and statistical significance will be set at $\mathrm{p}<0.05$. The primary outcome, mini-AHA scores at 12 months ca, will be compared between treatment groups using generalised linear models. The main effect entered will be treatment group (mCMIT/BIM), and stratification variables (age, gender, side) will be entered as covariables. The distributional family will be Gaussian and the identity link will be used. The secondary analyses will use similar methods to compare outcomes between groups at 24 months ca for bimanual coordination (AHA), cognitive development (domains of Bayley Scale of Infant Development III) and self-care domains of PEDI-CAT. For dichotomous outcomes, the distributional family will be binomial and the logit link will be used. Where continuous data exhibit skewness not overcome by transformation, non-parametric methods (for example the Mann-Whitney U Test or quantile regression) will be used. For $\mathrm{H}^{3},{ }^{3}$ magnitude of central changes between groups will be determined using quantitative analysis of dMRI statistical parametric maps. Data will be compared between groups using generalised estimating equations.

Validity of results will be checked using baseline and general descriptive information available for all eligible families. This includes comparing key characteristics of families who completed the study with those who enrolled in the study but did not complete and those who did not enrol. Multiple imputation techniques will be used if to adjust for differential drop-out if appropriate. Professor Robert Ware, lead biostatistician, will provide expert input for guiding and assisting with analysis.

\section{ETHICS AND DISSEMINATION}

This study protocol presents the background and design for a randomised comparison trial comparing intensive Baby CIMT training to the same dose of Baby BIM for children with congenital hemiplegia. The alternate approaches may impact neuroplasticity positively (balanced) or negatively (lateralised) which along with the duration of therapeutic responses will guide clinicians on choice of the most effective treatments. Our team has commenced the first implementation studies of intensive upper limb therapy in school-aged children with hemiplegia, ${ }^{78}$ and thus findings of this proposed study in young infants are able to be translated rapidly into clinical practice.

Full ethical approvals for this study have been obtained from the Children's Health Queensland Hospital and Health Service Human Research Ethics Committee (HREC/14/QRCH/376: ethical review provided for 20 sites: Lady Cilento Children's Hospital, Royal Brisbane and Women's Hospital, Mater Mothers' Hospital, Gold Coast University Hospital, Sunshine Coast University Hospital, Nambour General Hospital, The Children's Hospital at Westmead, Children's Hospital Randwick, Westmead Hospital, Royal North Shore Hospital, Liverpool Hospital, Nepean Hospital, Royal Prince Alfred Hospital, Royal Women's Hospital, St George Hospital, Blacktown Hospital, Campbelltown Hospital, John Hunter Children's Hospital, Royal Children's Hospital and Monash Medical Centre), Medical Ethics Committee of The University of Queensland (2015000013), Cerebral Palsy Alliance Ethics Committee (2015-01-02) and the Princess Margaret Hospital Human Research Ethics Committee (2015023EP: ethical review provided for two sites: Princess Margaret Hospital for Children and King Edward Memorial Hospital for Women).

To our knowledge, this study is the first to investigate and compare the effects of two approaches to very early upper limb rehabilitation for infants at high risk of congenital hemiplegia implemented by parents coached by therapists. Furthermore, we will be evaluating outcomes of the Baby CIMT versus Baby BIM training programmes across all relevant domains of the International Classification of Functioning, Disability and Health using the most valid and reliable assessment tools available for use. We anticipate that the results of this study will be disseminated through peer-reviewed journals and national and international academic conferences as well as our established clinical and consumer networks.

Author affiliations
${ }^{1}$ Queensland Cerebral Palsy and Rehabilitation Research Centre, Centre for
Children's Health Research, The University of Queensland, Brisbane, Australia
${ }^{2}$ Children's Allied Health Research, Children's Health Queensland, Brisbane,
Queensland, Australia
${ }^{3}$ School of Health and Rehabilitation Sciences, The University of Queensland,
Brisbane, Australia
${ }^{4}$ Cerebral Palsy Alliance, The University of Sydney, Sydney, Australia
${ }^{5}$ The Australian E-Health Research Centre, Health and Biosecurity, CSIRO, Brisbane,
Australia
${ }^{6}$ School of Occupational Therapy and Social Work, Curtin University, Perth, Australia


${ }^{7}$ Perth Children's Hospital, Perth, Western Australia, Australia

${ }^{8}$ Royal Children's Hospital, Melbourne, Australia

${ }^{9}$ IRCCS Stella Maris and The University of Pisa, Pisa, Italy

${ }^{10}$ School of Allied Health, Australian Catholic University, North Sydney, Australia

${ }^{11}$ Department of Women's and Children's Health, Neuropediatric Unit, Karolinska Institutet, Stockholm, Sweden

${ }^{12}$ Menzies Health Institute Queensland, Griffith University Gold Coast, Nathan, Australia

${ }^{13}$ CSIRO Mathematical and Information Sciences Biomedical Imaging Group, Australian e-Health Research Centre

Correction notice None.

Twitter None.

Acknowledgements The authors would like to thank A/Professor Lena KrumlindeSundholm from the Karolinska Institute who has advised on the Baby CIMT and Baby BIM content. The authors acknowledge A/Professor Karen Walker, Grace Centre for Newborn Care, The Children's Hospital at Westmead; Dr Russell Dale, Sydney Children's Hospital Network; Dr Roslyn Ward, School of Paediatrics and Child Health, The University of Western Australia, Perth; Dr Mary Sharp and Dr Noel French, Neonatologists, King Edward Memorial Hospital for Women, Perth for their roles in recruitment for the study. The authors would like to thank Dr Tracey Evans at the Queensland Cerebral Palsy and Rehabilitation Research Centre for her contributions to the overall management of the study. The authors would also like to thank Alana Muir for her role in evaluating the study fidelity. The authors would also like to thank the therapists who will undertake intervention in the REACH trial including Debra Khan, Danni Centorame, Emma Taylor and Nerida Taylor and Olivia Naylor.

Collaborators None.

Contributor No additional.

Contributors RNB is the chief investigator (Cl) and together with JZ, LS, IN, NB, KP, CE, SG, AG, KW, JV, CM, RW and SR designed, established and achieved funding for this research study. CM, MW, LF also contributed to study design. RNB, LS, CM, MW, SG and CE are responsible for ethics applications and reporting. RNB, LS, IN, CE and SG are responsible for recruitment. RNB, LS, IN, $\mathrm{CE}, \mathrm{SG}$ and $\mathrm{CM}$ supervise the data collection and implementation of the training programme. RNB, KP and SR were responsible for design and implementation of the Advanced Brain Imaging outcomes. RNB, JZ, LS, IN, NB, KP, CE, SG, AG, KW, $\mathrm{JV}, \mathrm{CM}, \mathrm{RW}$ will take lead roles on preparation for publications on the clinical outcomes of the study and RNB, SR, SF, KP and AG will take lead roles on the neuroscience publications from the study. RW will take on a lead role of the statistical analysis for the study. RB and the Cls drafted the final version of this manuscript. All authors contributed to the writing of this manuscript and have critically reviewed and approved the final version. All data from this study will be submitted to peer review journals.

Funding This protocol and randomised trial is funded by the Australian National Health and Medical Research Council (NHMRC) Project Grant for REACH 1059332; NHMRC Early Career Fellowship no.1090828 (LS); NHMRC Research Fellowship (RB) 1105038. This study is funded by the Australian National Health and Medical Research Council (NHMRC) for a project grant no 1078877. The NHMRC has provided people support for the following team members: a Research Fellowship (RB, 1105038), Early Career Fellowship (LS, No1090828, KW, № 631712).

Disclaimer None.

Competing interests None declared.

Patient consent Obtained.

Ethics approval Full ethical approvals for this study have been obtained from the Children's Health Queensland Hospital and Health Service Human Research Ethics Committee (HREC/14/QRCH/376: ethical review provided for 20 sites: Lady Cilento Children's Hospital, Royal Brisbane and Women's Hospital, Mater Mothers' Hospital, Gold Coast University Hospital, Sunshine Coast University Hospital, Nambour General Hospital, The Children's Hospital at Westmead, Children's Hospital Randwick, Westmead Hospital, Royal North Shore Hospital, Liverpool Hospital, Nepean Hospital, Royal Prince Alfred Hospital, Royal Women's Hospital, St George Hospital, Blacktown Hospital, Campbelltown Hospital, John Hunter Children's Hospital, Royal Children's Hospital and Monash Medical Centre), Medical Ethics Committee of The University of Queensland (2015000013), Cerebral Palsy Alliance Ethics Committee (2015-01-02) and the Princess Margaret Hospital Human Research Ethics Committee (2015023EP: ethical review provided for two sites:
Princess Margaret Hospital for Children and King Edward Memorial Hospital for Women).

Provenance and peer review Not commissioned; externally peer reviewed.

Data sharing statement The funding bodies have no role in the study design, data collection, management, analysis and interpretation of data; writing of the report; and the decision to submit the reports for publication. The authors have ultimate authority over these activities. There is no additional unpublished data from this study to be shared.

Open Access This is an Open Access article distributed in accordance with the Creative Commons Attribution Non Commercial (CC BY-NC 4.0) license, which permits others to distribute, remix, adapt, build upon this work non-commercially, and license their derivative works on different terms, provided the original work is properly cited and the use is non-commercial. See: http://creativecommons.org/ licenses/by-nc/4.0/

(c) Article author(s) (or their employer(s) unless otherwise stated in the text of the article) 2017. All rights reserved. No commercial use is permitted unless otherwise expressly granted.

\section{REFERENCES}

1. Stanley F, Blair E. Cerebral palsies: epidemiology and causal pathways: MacKeith Press, 200.

2. Access Economics. The economic impact of cerebral palsy in Australia in 2007. Canberra 2008.

3. Sakzewski L, Ziviani J, Boyd R. Efficacy of upper limb interventions for children with unilateral cerebral palsy: systematic review and meta-analysis update. Pediatrics 2014;133:e175-204.

4. Eliasson AC. Bimanual training for children with unilateral $\mathrm{CP}-$ is this something new? Dev Med Child Neurol 2007;49:806.

5. Eliasson AC, Krumlinde-sundholm L, Shaw K, et al. Effects of constraint-induced movement therapy in young children with hemiplegic cerebral palsy: an adapted model. Dev Med Child Neurol 2005;47:266-75

6. Cobos-Carbó A, Augustovski F. CONSORT 2010 declaration: updated guideline for reporting parallel group randomised trials. Med Clin 2011;137:213-5.

7. ACPR Group. Report of the Australian Cerebral Palsy Register, Birth years 1993-2006, 2013.

8. Sakzewski L, Ziviani J, Abbott DF, et al. Randomized trial of constraint-induced movement therapy and bimanual training on activity outcomes for children with congenital hemiplegia. Dev Med Child Neurol 2011;53:313-20.

9. Al-Oraibi S, Eliasson AC. Implementation of constraint-induced movement therapy for young children with unilateral cerebral palsy in Jordan: a home-based model. Disabil Rehabil 2011;33:2006-12.

10. Eyre JA, Smith M, Dabydeen L, et al. Is hemiplegic cerebral palsy equivalent to amblyopia of the corticospinal system? Ann Neurol 2007;62:493-503.

11. Staudt M, Gerloff C, Grodd W, et al. Reorganization in congenital hemiparesis acquired at different gestational ages. Ann Neurol 2004;56:854-63.

12. Cramer SC, Sur M, Dobkin BH, et al. Harnessing neuroplasticity for clinical applications. Brain 2011;134:1591-609.

13. Guzzetta A, Pizzardi A, D'Acunto M, et al. Early development of hand motor function in infants with neonatal cerebral infarction. Dev Med Child Neurol 2009;51:78.

14. Thickbroom GW, Byrnes ML, Archer SA, et al. Differences in sensory and motor cortical organization following brain injury early in life. Ann Neurol 2001:49:320-7.

15. Boyd R, Perez M, Guzzetta A. Very early upper limb intervention for infants with asymmetric brain injury. Shepherd R, ed. Cerebral palsy in Infancy and Early childhood,. Section 4, specific methods of Motor training for infants. 1st ed. London: Elsevier, 2013.

16. Friel K, Chakrabarty S, Kuo HC, et al. Using motor behavior during an early critical period to restore skilled limb movement after damage to the corticospinal system during development. J Neurosci 2012;32:9265-76.

17. Martin JH, Chakrabarty S, Friel KM. Harnessing activity-dependent plasticity to repair the damaged corticospinal tract in an animal model of cerebral palsy. Dev Med Child Neurol 2011;53:9-13.

18. Rose S, Guzzetta A, Pannek K, et al. MRI structural connectivity, disruption of primary sensorimotor pathways, and hand function in cerebral palsy. Brain Connect 2011;1:309-16.

19. Krägeloh-Mann I, Horber V. The role of magnetic resonance imaging in elucidating the pathogenesis of cerebral palsy: a systematic review. Dev Med Child Neurol 2007;49:144-51. 
20. Basu AP, Pearse J, Kelly S, et al. Early intervention to improve hand function in hemiplegic cerebral palsy. Front Neurol 2015;281:281.

21. Chan AW, Tetzlaff JM, Altman DG, et al. SPIRIT 2013 statement: defining standard protocol items for clinical trials. Ann Intern Med 2013;158:200-7.

22. Haataja L, Mercuri E, Regev R, et al. Optimality score for the neurologic examination of the infant at 12 and 18 months of age. $J$ Pediatr 1999;135:153-61.

23. Romeo DM, Cioni M, Palermo F, et al. Neurological assessment in infants discharged from a neonatal intensive care unit. neurological assessment in infants discharged from a neonatal intensive care unit. Eur J Paediatr Neurol 2013;17:192-8.

24. Romeo DM, Ricci D, Brogna C, et al. Use of the hammersmith infant neurological examination in infants with cerebral palsy: a critical review of the literature. Dev Med Child Neurol 2015;58.

25. Romeo DM, Cioni M, Scoto M, et al. Neuromotor development in infants with cerebral palsy investigated by the Hammersmith Infant neurological examination during the first year of age. Eur $\mathrm{J}$ Paediat Neurol 2008;12:24-31.

26. Fiori S, Cioni G, Klingels K, et al. Reliability of a novel semiquantitative scale for classification of brain MRI for children with cerebral palsy. Dev Med Child Neurol 2014;56:839-45.

27. Eliasson AC, Sjöstrand L, Ek L, et al. Efficacy of baby-CIMT: study protocol for a randomised controlled trial on infants below age 12 months, with clinical signs of unilateral CP. BMC Pediatr 2014;14:141

28. Eliasson AC, Shaw K, Berg E, et al. An ecological approach of constraint induced movement therapy for 2-3-year-old children: a randomized control trial. Res Dev Disabil 2011;32:2820-8.

29. Eliasson AC, Krumlinde-Sundholm L, Gordon AM, et al. Guidelines for future research in constraint-induced movement therapy for children with unilateral cerebral palsy: an expert consensus. Dev Med Child Neurol 2014;56:125-37.

30. Fagard J, Jacquet A-Y. Onset of bimanual coordination and symmetry versus asymmetry of movement. Infant Behavior and Development 1989;12:229-35.

31. Case-Smith J. Hand skill development in the context of infants' play: birth to 2 years. Pehoski AHC, ed. Hand function in the Child: foundations for Remediation. St Louis: MO: Mosby, 2005:117-41.

32. Krumlinde-Sundholm L, Ek L, Eliasson AC. What assessments evaluate use of hands in infants? A literature review. Dev Med Child Neurol 2015;57(Suppl 2):37-41.

33. Greaves S, Imms C, Krumlinde-Sundholm L, et al. Bimanual behaviours in children aged 8-18 months: a literature review to select toys that elicit the use of two hands. Res Dev Disabil 2012;33:240-50.

34. Novak I, Cusick A. Home programmes in paediatric occupational therapy for children with cerebral palsy: where to start? Aust Occup Ther J 2006:53:251-64.

35. An M, Palisano RJ. Family-professional collaboration in pediatric rehabilitation: a practice model. Disabil Rehabil 2014;36:434-40.

36. Graham F, Rodger S, Ziviani J. Coaching parents to enable children's participation: an approach for working with parents and their children. Aust Occup Ther J 2009:56:16-23.

37. Baldwin P, King G, Evans J, et al. Solution-focused coaching in pediatric rehabilitation: an integrated model for practice. Phys Occup Ther Pediatr 2013;33:467-83.

38. Soska KC, Galeon MA, Adolph KE. On the other hand: overflow movements of infants' hands and legs during unimanual object exploration. Dev Psychobiol 2012;54:372-82.

39. Fallang B, Saugstad OD, Hadders-Algra M. Goal directed reaching and postural control in supine position in healthy infants. Behav Brain Res 2000;115:9-18.

40. Soska KC, Adolph KE, Johnson SP. Systems in development: motor skill acquisition facilitates three-dimensional object completion. Dev Psychol 2010;46:129-38.

41. Biringen Z, Derscheid D, Vliegen N, et al. Emotional availability (EA): theoretical background, empirical research using the EA scales, and clinical applications. Developmental Review 2014;34:114-67.

42. Eakes GG, Burke ML, Hainsworth MA, et al. Middle-range theory of chronic sorrow. Image J Nurs Sch 1998;30:179-84.

43. Hayes SC, et al. Acceptance and Commitment therapy: an experiential approach to behavior change. New York: Guilford Press, 2003.

44. Bellg AJ, Borrelli B, Resnick B, et al. Enhancing treatment fidelity in health behavior change studies: best practices and recommendations from the NIH Behavior Change Consortium. Health Psychol 2004:23:443-51.

45. Poltawski L, Norris M, Dean S. Intervention fidelity: developing an experience-based model for rehabilitation research. J Rehabil Med 2014:46:609-15.
46. Toomey E, Matthews J, Guerin S, et al. Development of a feasible implementation fidelity protocol within a complex physical therapyled self-management intervention. Phys Ther 2016;96:1287-98.

47. Hoffmann TC, Glasziou PP, Boutron I, et al. Better reporting of interventions: template for intervention description and replication (TIDieR) checklist and guide. BMJ 2014;348:g1687.

48. Lovibond PF, Lovibond SH. The structure of negative emotional states: comparison of the depression anxiety stress scales (DASS) with the Beck Depression and anxiety inventories. Behav Res Ther 1995;33:335-43.

49. Vliegen N, Luyten P, Biringen Z. A multimethod perspective on emotional availability in the postpartum period. Parenting 2009;9:228-43

50. Kidokoro H, Anderson PJ, Doyle LW, et al. Brain injury and altered brain growth in preterm infants: predictors and prognosis. Pediatrics 2014;134:e444-e453.

51. Woodward LJ, Anderson PJ, Austin NC, et al. Neonatal MRI to predict neurodevelopmental outcomes in preterm infants. $N$ Engl $J$ Med 2006;355:685-94.

52. Nguyen The Tich S, Anderson PJ, Shimony JS, et al. A novel quantitative simple brain metric using MR imaging for preterm infants. AJNR Am J Neuroradiol 2009;30:125-31.

53. Spittle AJ, Doyle LW, Anderson PJ, et al. Reduced cerebellar diameter in very preterm infants with abnormal general movements. Early Hum Dev 2010;86:1-5.

54. Kidokoro H, Neil JJ, Inder TE. New MR imaging assessment tool to define brain abnormalities in very preterm infants at term. AJNR Am J Neuroradiol 2013;34:2208-14.

55. , . In: Einspieler C, Prechtl H, Bos A, Ferrari F, Cioni G, et al. eds. Precht/'s method of qualitative assessment of general movements in preterm, term and young infants. London: Mac Keith Press, 2004.

56. Prechtl HF, Einspieler C, Cioni G, et al. An early marker for neurological deficits after perinatal brain lesions. Lancet 1997;349:1361-3.

57. Guzzetta A, Pizzardi A, Belmonti V, et al. Hand movements at 3 months predict later hemiplegia in term infants with neonatal cerebral infarction. Dev Med Child Neurol 2010;52:767-72.

58. Krumlinde-Sundholm L, Ek L, Guzzetta A, et al. The Hand Assessment for Infants, a new test for measuring use of hands and possible asymmetry in infants 3-10 months of age. Developmental Medicine and Child Neurology 2015;57:54-5.

59. Guzzetta A, Boyd RN, Perez M, et al. UP-BEAT (Upper Limb Baby Early Action-observation training): protocol of two parallel randomised controlled trials of action-observation training for typically developing infants and infants with asymmetric brain lesions. BMJ Open 2013;3:e002512.

60. Eliasson AC, Krumlinde-Sundholm L, Rösblad B, et al. The Manual Ability classification system (MACS) for children with cerebral palsy: scale development and evidence of validity and reliability. Dev Med Child Neurol 2006;48:549-54.

61. Eliasson AC, Ullenhag A, Wahlström U, et al. Mini-MACS: development of the Manual Ability classification system for children younger than 4 years of age with signs of cerebral palsy. Dev Med Child Neurol 2017;59:72-8.

62. Palisano R, Rosenbaum P, Walter S, et al. Development and reliability of a system to classify gross motor function in children with cerebral palsy. Dev Med Child Neurol 1997;39:214-23.

63. Palisano RJ, Cameron D, Rosenbaum PL, et al. Stability of the gross motor function classification system. Dev Med Child Neurol 2006;48:424-8.

64. Wood E, Rosenbaum P. The gross motor function classification system for cerebral palsy: a study of reliability and stability over time. Dev Med Child Neurol 2000;42:292-6.

65. Gorter JW, Rosenbaum PL, Hanna SE, et al. Limb distribution, motor impairment, and functional classification of cerebral palsy. Dev Med Child Neurol 2004;46:461-7.

66. Surveillance of Cerebral Palsy in Europe registers. Surveillance of Cerebral Palsy in Europe: a Collaboration of Cerebral Palsy surveys and registers. Dev Med Child Neurol 2000;42:816-24.

67. Fiori S, Cioni G, Klingels K, et al. Reliability of a novel semiquantitative scale for classification of brain MRI for children with cerebral palsy. Developmental Medicine and Child Neurology 2014;56:839-45.

68. Ashwal S, Russman BS, Blasco PA, et al. Practice parameter: diagnostic assessment of the child with cerebral palsy: report of the Quality Standards Subcommittee of the American Academy of Neurology and the Practice Committee of the Child Neurology Society. Neurology 2004;62:851-63.

69. Fiori S, Guzzetta A, Pannek K, et al. Validity of semiquantitative scale for brain MRI in unilateral cerebral palsy due to periventricular white matter lesions: relationship with hand 
sensorimotor function and structural connectivity. Neuroimage Clin 2015;8:104-9.

70. Greaves S, Imms C, Dodd K, et al. Development of the MiniAssisting hand Assessment: validation of the play session and item generation.. 20135510301037.

71. Holmefur M, Krumlinde-Sundholm L, Bergström J, et al. Longitudinal development of hand function in children with unilateral cerebral palsy. Dev Med Child Neurol 2010;52:413-21.

72. Bayley N. Bayley Scales of Infant and Toddler Development Third Edition. San Antonio: The Psychological Corporation, 2005.

73. Albers CA, Grieve AJ. Bayley scales of infant and toddler development, third edition. J Psychoeduc Assess 2007;25:180-90.

74. Hayley S, Coster W, Ludlow L. Pediatric evaluation of disability inventory: clinical interpretation of summary scores using rasch rating scale methyodology. Phys Med Rehabil Clin N Am 1993;4:529-40.

75. Tournier J, Calamante F, Connelly A. MRtrix: diffusion tractography in crossing fiber regions. Int J Imaging Syst Technol 2012;22:53-66.

76. Pannek K, Guzzetta A, Colditz PB, et al. Diffusion MRI of the neonate brain: acquisition, processing and analysis techniques. Pediatr Radiol 2012;42:1169-82.

77. Holmefur M, Aarts P, Hoare B, et al. Retest and alternate forms reliability of the Assisting Hand Assessment (PhD Thesis). Stockholm: Karolinska Institutet, 2009.

78. Sakzewski L, Ziviani J, Boyd RN. Translating evidence to increase Quality and Dose of Upper Limb therapy for children with unilateral cerebral palsy: a pilot study. Phys Occup Ther Pediatr 2016;36:305-29. 